\section{Starting on prevention in practice}

Prevention is supposed to be everybody's business, but often it is nobody's. Doctors are too busy with lengthening queues of patients demanding curative medicine; the Government is preoccupied with the shortage of money; the newspapers and television revel in the sensational; and individuals, living in the shadow of unemployment, the recession, and the bomb, are thinking short-term and are unimpressed by the exhortations of preventive zealots. Thus the Royal College of General Practitioners is to be congratulated on its attempts to consider practically how more preventive medicine can be introduced into general practice. Five college working parties have been considering these questions, and three have now reported. ${ }^{1-3}$

Most of the great successes in raising health standards have been preventive. Antenatal care and immunisation, which are both now routine matters undertaken by most general practitioners, have saved many lives, and we have good evidence that doctors can do more. Seeking out and treating hypertensive patients can reduce mortality ${ }^{4}$; and general practitioners can with simple measures help people to stop smoking. ${ }^{5}$ As the report on the prevention of arterial disease says: "Probably about a half of all strokes and a quarter of all coronary deaths under 70" are preventable.

The reports focus well the concept of prevention: they discuss the evidence for various kinds of prevention and list in detail what a general practitioner can do. Anything a doctor chooses to do, however, will have to be done in addition to what he does now; there is no evidence that time spent on prevention will mean a diminution in the normal work load. General practitioners can undertake prevention in several different ways: they can devote a little of each consultation to prevention, they can organise practice-based schemes, and they can work within the local community. Because time, energy, and resources are limited it will be crucial to experiment with different methods and to audit what is achieved. Much of the work, as the reports point out, can be delegated, and they suggest that principals should be allowed to appoint up to two more staff to do preventive work.

Unusually for reports from the Royal College of General Practitioners, much is said about money and incentives. Little will be achieved, the main report argues, if general practitioners are not paid to do more preventive work. It may seem paradoxical that the working party should argue, firstly, that "anticipatory care is the main direction of growth for the primary health services in the foreseeable future," and then say that nothing will happen unless doctors are given financial incentives. But it shows a realistic approach to the problem. "Anticipatory care" is a phrase that the main working party has coined to emphasise the indivisibility of prevention, diagnosis, and care. The working party does not seem to like the phrase much itself, however, and throughout the report refers much more to prevention than to "anticipatory medicine." New phrases more often confuse than clarify, and there seems no need for this one. Another practical problem is that no matter how successful preventive medicine proves to be, everybody will succumb to some disease eventually and need medical care. So is it ever conceivable that prevention will reduce a doctor's work load?

These reports no doubt reflect the thinking and enthusiasm of the current college leaders, who are to be congratulated on their initiative. For if all else fades away the lists of diseases amenable to prevention and of preventive topics for audit could be useful on a daily basis to general practitioners everywhere.

${ }^{1}$ Main Working Party appointed by the Council of the Royal College of General Practitioners. Health and prevention in primary care. London RCGP, 1981.

2 Arterial Disease Working Party appointed by the Council of the Royal College of General Practitioners. Prevention of arterial disease in general practice. London: RCGP, 1981.

3 Preventive Psychiatry Working Party appointed by the Council of the Royal College of General Practitioners. Prevention of psychiatric disorders in general practice. London: RCGP, 1981.

${ }^{4}$ Hypertension Detection and Follow-up Co-operative Group. Five-year findings of the hypertension detection and follow-up programme: 1 reduction in mortality of persons with high blood pressure, including mild hypertension. $\mathcal{F} A M A$ 1979;242:2562-71.

${ }^{5}$ Russell MAH, Wilson C, Taylor C, Baker CD. Effect of general practitioners' advice against smoking. $\mathrm{Br}$ Med f 1979;ii :231-5.

\section{Second remissions in child- hood acute lymphoblastic leukaemia}

In the proliferation of terms that have come to surround the management of cancer, "complete remission" is one that has considerable prognostic importance. This term is applied essentially to an artefact in that it describes the eradication of all disease detectable by the techniques at our disposal. In the acute leukaemias the difficulty of differentiating between normal and abnormal cells has been acknowledged by conceding that a small percentage of abnormal blast cells in the bone marrow is consistent with a complete remission. Newer techniques, both cytochemical and those that enable the surface characteristics of cells to be screened individually, have increased the sensitivity of the endpoint of complete remission in some conditions. These factors alone emphasise the need for its redefinition.

Even in its crudest definition, however, complete remission in the acute leukaemias, cancer of the ovary, small-cell lung cancer, the teratomas, the lymphomas, and some malignancies of childhood is associated with a better quality of life and longer survival than are seen in the patients who do not achieve it. Complete remission is frequently used as an indicator of the effectiveness of treatment; but this may be inappropriate, for it may reflect the natural sensitivity of a biological variant of the disease to a standard form of treatment, rather than the impact of that treatment on a standard population. Unfortunately, few presenting characteristics enable us to predict who will achieve complete remission. Even in those conditions such as acute lymphoblastic leukaemia and the teratomas, where chemotherapy may result in a "complete remission" with a total eradication of the neoplastic process, this eradication is established only when the patient dies of a condition other than his original malignancy - though clearly this becomes more likely with time.

Once relapse of childhood acute lymphoblastic leukaemia has occurred, a subsequent complete remission is widely held to have less prognostic importance. A review by Cornbleet and Chessells ${ }^{1}$ of 53 children having bone marrow relapse during 1972-7 supports this view. Whereas 15 out of 16 who had completed and subsequently discontinued maintenance treatment after two to three years achieved complete remission after relapse, only 25 out of 37 who were still receiving treatment at the time of relapse did so. The median survival of the two groups was 72 and 37 weeks, with no long-term survivors in the latter group and four-fifths of all these patients dying 